\title{
Design and real-time test of a hybrid energy storage system in the microgrid with the benefit of improving the battery lifetime
}

\author{
Jianwei $\mathrm{Li}^{\mathrm{a}}{ }^{\mathrm{b}}$, Rui Xiong ${ }^{\mathrm{a}, *}$, Hao $\mathrm{Mu}^{\mathrm{a}, *}$, Bertrand Cornélusse ${ }^{\mathrm{b}}$, Philippe Vanderbemden ${ }^{\mathrm{b}}$, \\ Damien Ernst ${ }^{\mathrm{b}}$, Weijia Yuan ${ }^{\mathrm{c}}$ \\ ${ }^{a}$ National Engineering Laboratory for Electric Vehicles, School of Mechanical Engineering, Beijing Institute of Technology, Beijing 100081, China \\ ${ }^{\mathrm{b}}$ University of Liege, Institut Montefiore, Liege 4000, Belgium \\ ${ }^{c}$ University of Bath, Department of Electronic and Electrical Engineering, Bath, UK
}

\section{H I G H L I G H T S}

- A new control method of the hybrid energy storage system is introduced.

- The battery lifetime has been proved to be extended in the real-time experiment.

- A new experiment method is proposed using the RTDS\&HIL to give real-time verification.

- A battery lifetime prediction method is introduced.

- The RTDS\&HIL scheme highlights a flexible real-time experimental approach.

\section{A R T I C L E I N F O}

\section{Keywords:}

Battery

Battery lifetime extension

Hybrid energy storage system

Hardware in the loop

Real-time digital simulator

\begin{abstract}
A B S T R A C T
This study proposes a hybrid energy storage system (HESS) composed of the superconducting energy storage system (SMES) and the battery. The system is designed to compensate power fluctuations within a microgrid. A novel control method is developed to share the instantaneous power between the SMES and the battery. The new control scheme takes into account the characteristics of the components of the HESS, and the battery charges and discharges as a function of the SMES current rather than directly to the power disturbances. In this way, the battery is protected from the abrupt power changes and works as an energy buffer to the SMES. An new hardware-in-loop experiment approach is introduced by integrating a real-time digital simulator (RTDS) with a control circuit to verify the proposed hybrid scheme and the new control method. This paper also presents a battery lifetime prediction method to quantify the benefits of the HESS in the microgrid. A much better power sharing between the SMES and the battery can be observed from the experimental results with the new control method. Moreover, compared to the battery only system the battery lifetime is quantifiably increased from 6.38 years to 9.21 years.
\end{abstract}

\section{Introduction}

The microgrid concept, that is defined as a low-voltage system having a cluster of loads and generators capable of providing the stable electricity to the localised area, is regarded as an effective system formation to enhance the renewable power penetrations [1-3]. Due to the variable nature of renewables, the generated power profile may not be able to match the load requirement. Accordingly, much attention has been focused on the development of energy storage technologies to compensate the power disturbances and maintain the system stability [4-6].
The battery storage system (BSS) which has a relatively high level of maturity was reported to be used in the microgrid by many previous works [7-9]. A BSS has a relatively high energy density and a high efficiency [10-13], which makes it an effective method to tackle the power balancing issues in a microgrid [14]. Nevertheless, a BSS faces two challenges. First, the service lifetime of the battery is limited. Depending on the variable nature of the renewable energy source, the battery in a microgrid may experience many short-term charge/discharge cycles. Secondly, compared to short-term energy storage technologies such as a SMES, the power density of the battery is much lower, which makes it difficult for the battery to handle the high-

\footnotetext{
* Corresponding authors.

E-mail addresses: rxiong@bit.edu.cn (R. Xiong), muhao_922@bit.edu.cn (H. Mu), w.yuan@bath.ac.uk (W. Yuan).
} 
frequency fluctuations. The SMES is characterised by an outstanding power density and is able to response to the power requirements very quickly $[2,15-18]$. However, the energy density of the SMES is much lower than that of the battery [19]. The concept of the SMES/battery hybrid energy storage is, therefore, proposed by combining two kinds of complementary energy storages. In this paper, a detailed scheme of the SMES/battery hybrid energy storage is presented, which has the advantages of both primary energy storage systems meanwhile complementing the disadvantages of each ESS.

The control of the battery and the SMES is the key factor to achieve the expected power distributions and complementary functions of the ESSs [20]. For the single energy storage technology used in the power system, the charge/discharge demands for the single ESS is straightforward [21]. However, in the hybrid energy storage scheme, the control task is much more complicated because the control needs to effectively combine the harmonious operation of two storage technologies such that they complement each other [22-24]. Fuzzy control, which can realize power management in nonlinear systems without accurate system modelling has been proven highly suitable for coordination of multiple energy sources [25]. Ise et al. [26] propose a fuzzy control based method in railway power systems, achieving effective power sharing between the battery and the SMES. However, some specific constraints and fuzzy regions used in this control are selected empirically, which sometimes may lead to sub-optimal design choices. Wang et al. in [27] proposed the conceptual control method that classifies the power requirements manually and distributes the power demands to the different energy storage systems based on their classification. However, the accuracy of this controller is highly dependent on the specific implementation. The filter based power control method which uses the inherent filtration characteristic of the SMES or supercapacitor to allocate low-frequency charge cycling to the battery has been applied in EVs [27-29] and renewable generations [30,31]. In this control scheme, the SMES and the battery are in parallel position and deal with the power fluctuation at the same time. Consequently, the battery may still experience the high-frequency power fluctuations which result in stinging charge/discharge of the battery. A modified fraction control method is, therefore, developed to share the power between the SMES and the battery. In the new method, the SMES and the battery are in series position, and the power disturbances are firstly dealt by the SMES. The battery works as the energy buffer to maintain the SMES current. Hence, the battery charges and discharges according to the SMES current rather than the instantaneous net power. The experiment shows that compared with the preceding fraction based HESS control, the new control scheme is able to protect the battery from abrupt power changes.

Generally, it is difficult to test the new control scheme used in the power system in the real experiments $[32,33]$. Hence, it comes to another novelty of this work that introduces a RTDS and HIL experimental to verify the hybrid design in a power system level. The RTDS which has real-time computing capability, is regarded as a very effective tool for the experimental verification of power systems. Also, the signals and measurements in the RTDS are real-time data, which make it possible to interface with the external hardware/devices. By adopting these advantages, an external circuit consisting of the digital signal processor (DSP) and the analogue/digital interfaces, is integrated with the RTDS forming a HIL test system.

Additionally, batteries have a significant impact on the budget when taking the whole life cost, replacement and maintenance into account. Hence, the quantitative analysis of the battery lifetime improvement is an essential process to evaluate the effectiveness of the SMES/battery HESS. A battery lifetime prediction method is also introduced in this study. The results show that the battery lifetime is predicted as 6.38 years in the battery only system, whereas in the same application the battery service time can be improved to 9.21 years by using the SMES/battery hybrid scheme.



Fig. 1. The system configuration.

\section{Methodology}

\subsection{System description}

As shown in Fig. 1, a microgrid system based on the benchmark scheme [2,34] with renewable generations and the SMES/battery HESS, is established in the RTDS. The battery model in the RSCAD which works a controlled voltage source with a series resistor is used in this paper. Since the battery is protected from the short-term frequency charge/discharge processes in this study, so the simple model in the RDCAD software is good to reflect/count the decrease of the short-term frequency charge/discharge cycles. The SMES and the battery are modelled using the methods presented in [31]. Tow DC/DC converters and an AC/DC converter are used to interface the energy storage systems into the AC bus. The converters are modelled using the small-timestep model in the RSCAD software. The hybrid energy storage control algorithm is implanted in the external circuit using a DSP TMS320F28335. A more detail introduction of this RTDS\&HIL test system is presented in Section 3.

\subsection{Control method}

The system control scheme consists of two parts: the voltage source control (VSC) of the AC/DC converter and the new power sharing control of the DC/DC converters is shown in Fig. 2. In this scheme, the HESS is modelled as the voltage source to compensate the system power fluctuations.

\subsubsection{Voltage source control}

In a grid connected microgrid system, the energy storage units could get the power and active power reference from the main grid $[35,36]$. However, in the off-grid system, there are no power and active power references $[29,37,38]$. Hence, as shown in Fig. 2, the current references in d-q axis $\left(I_{d_{-} r e f}\right.$ and $\left.I_{q_{-} r e f}\right)$ is generated based on the instantaneous 




Fig. 2. The hybrid energy storage control scheme.
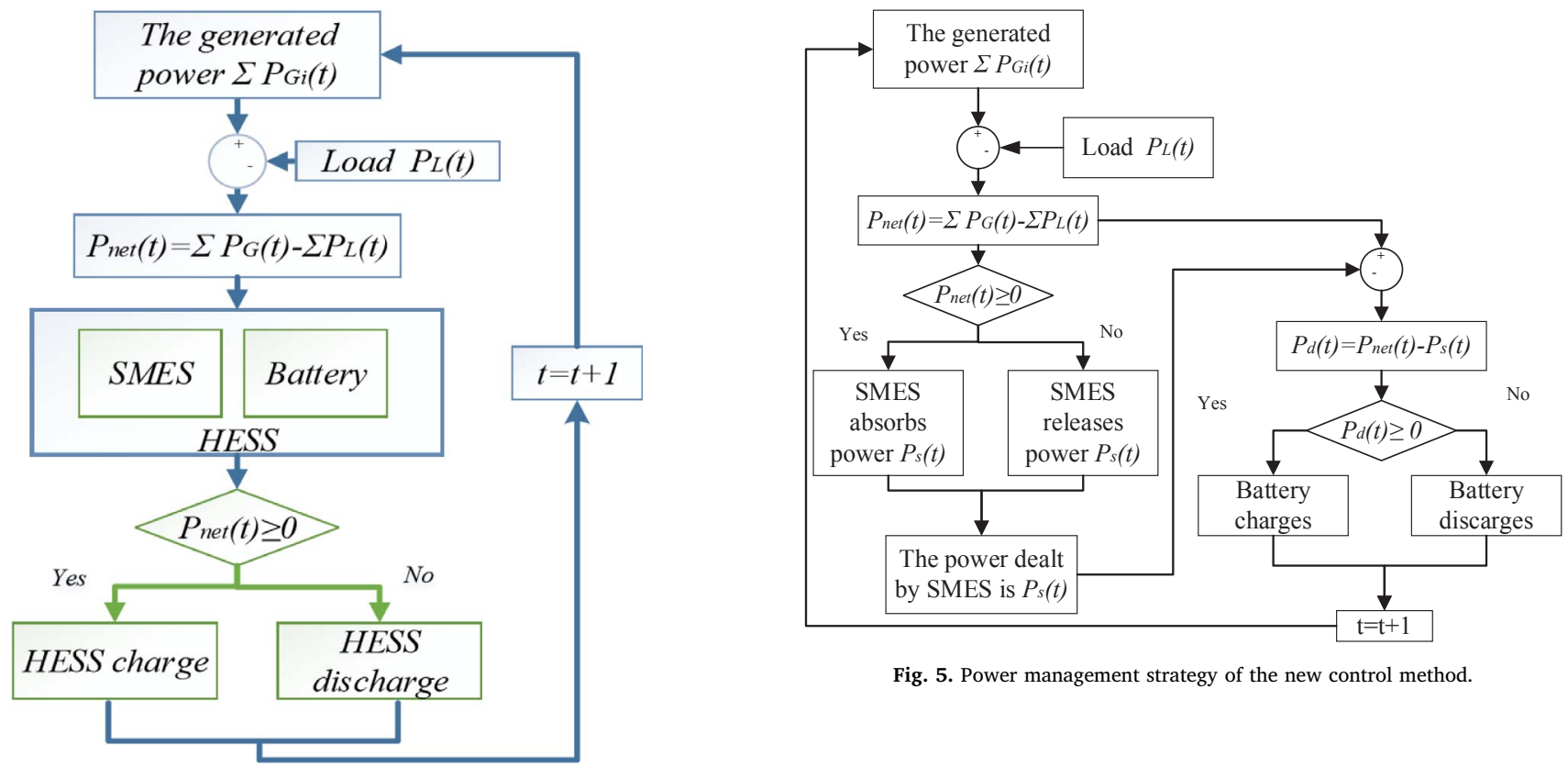

Fig. 5. Power management strategy of the new control method.

Fig. 3. Power management strategy of the filtration control.

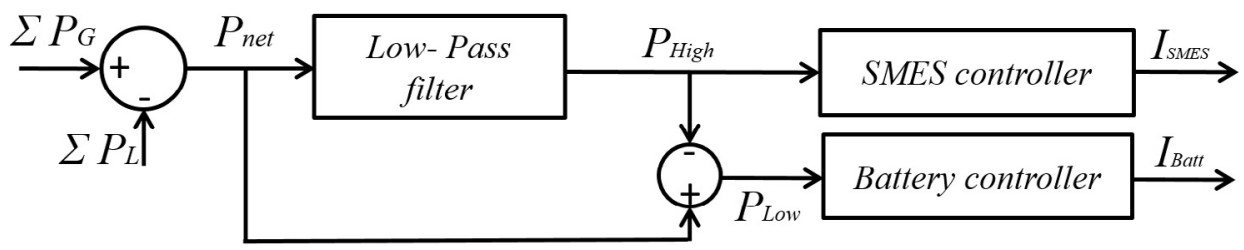

Fig. 4. The filtration based HESS control method. 




Fig. 6. The new priority-based power sharing control.

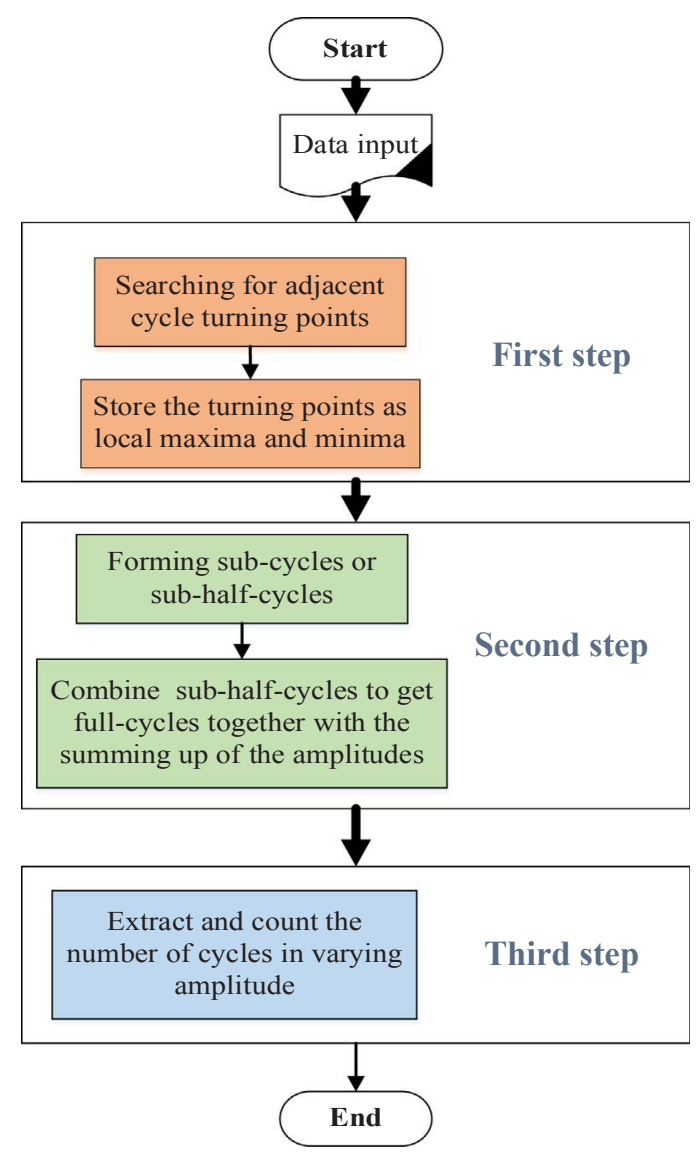

Fig. 7. The rain-flow cycle-counting algorithm.

measurement and desired values of the frequency and line voltage at the AC bus. Then, the current references $I_{d_{-} r e f}$ and $I_{q_{-} \text {ref }}$ obtained from the external control are transferred to the internal control which is used to give the reference voltage at the AC side of the AC/DC. Once the voltage references d-q axis $V_{d_{-} r e f}$ and $V_{q_{-} \text {ref }}$ are determined, the $d q$ to $a b c$ transformation block is used to transform the $V_{d_{-} r e f}$ and $V_{q_{-} r e f}$ to the three phase voltage references for the AC/DC converter. The phase-lock loop (PLL) is implemented to give the reference phase angle for the ad/ abc transformation. The desired three phase voltages are compared with the triangular to generate the PWM signals for the AC/DC converter.

\subsection{2. $D C / D C$ control}

(a) Previous filtration control
As shown in Fig. 3, the power management strategy of the previous filtration control is not complicated. The SMES and the battery are in parallel position and come into action at the same time to compensate the instantaneous power fluctuations. The SMES has higher power capacity and faster charge/discharge speed hence is more sensitive to the high-frequency power than the battery. As a result, to some extent, the battery is protected from the frequent charge and discharge.

The filtration based hybrid control method is illustrated in Fig. 4. By taking advantage of the low-pass filtering characteristics of the SMES, the net power can be arranged between the SMES and the battery. However, as it can be seen from this method, the operation reference for both the battery and SMES is the instantaneous net power, which means the ESSs response to the power disturbance at the same time. Any abrupt power changes could still be seen by the battery. Therefore, the battery may undergo abrupt current changes which will cause battery lifetime degradation. The SMES has almost unlimited charge/ discharge cycles and high current handling capability which make it able to respond to any requirements without capacity degradation. Therefore, a modified control method is proposed in this paper.

(b) The new priority-based power sharing control

The modified control method is designed based on the power management strategy illustrated in Fig. 5. As shown in Fig. 5, given the varying load power requirements, instantaneous net power can be obtained: $P_{\text {net }}(t)=\sum P_{G}(t)-\sum P_{L}(t)$. The SMES will come into action depending on the value of the net power: if $P_{n e t}(t)>0$, it means the generated power is greater than load demand and the SMES will be charged, otherwise the SMES will discharge. Assuming the power dealt by SMES is $P_{S}(t)$, then it obtains the power deficiency $P_{d}(t)=P_{\text {net }}(t)-P_{s}(t)$ and the battery is expected to handle this amount of power. In this way, the SMES is fully active to the power disturbance and the energy of the SMES is buffered by the battery.

To achieve the power management strategy shown in Fig. 5, the modified control method for the HESS is developed as shown in Fig. 6.

The power difference between the generation $P G$ and the load demand $P L$ as shown in Fig. 6, will cause the voltage fluctuation of the DC bus. To maintain the DC bus voltage Vbus to the desired range, the hybrid energy storage system needs to charge/discharge the current IH. As it can be seen from the Fig. 6, in the new control method, the power disturbances is dealt firstly, by the SMES by tracking the current IH. Then, the SMES current is maintained at the reference value ISMES_ref with the help of the battery. The ISMES_ref is the SMES reference current witch is selected based on the system margin and the maximal SMES current. The battery charge/discharge to track the SMES current, which means the battery works as energy storage devices for the SMES rather than directly for the microgrid system. Due to the inductive characteristic of the SMES coil, the current flowing the SMES cannot change abruptly. Hence, no abrupt power changes can be seen by the battery. 
(a)

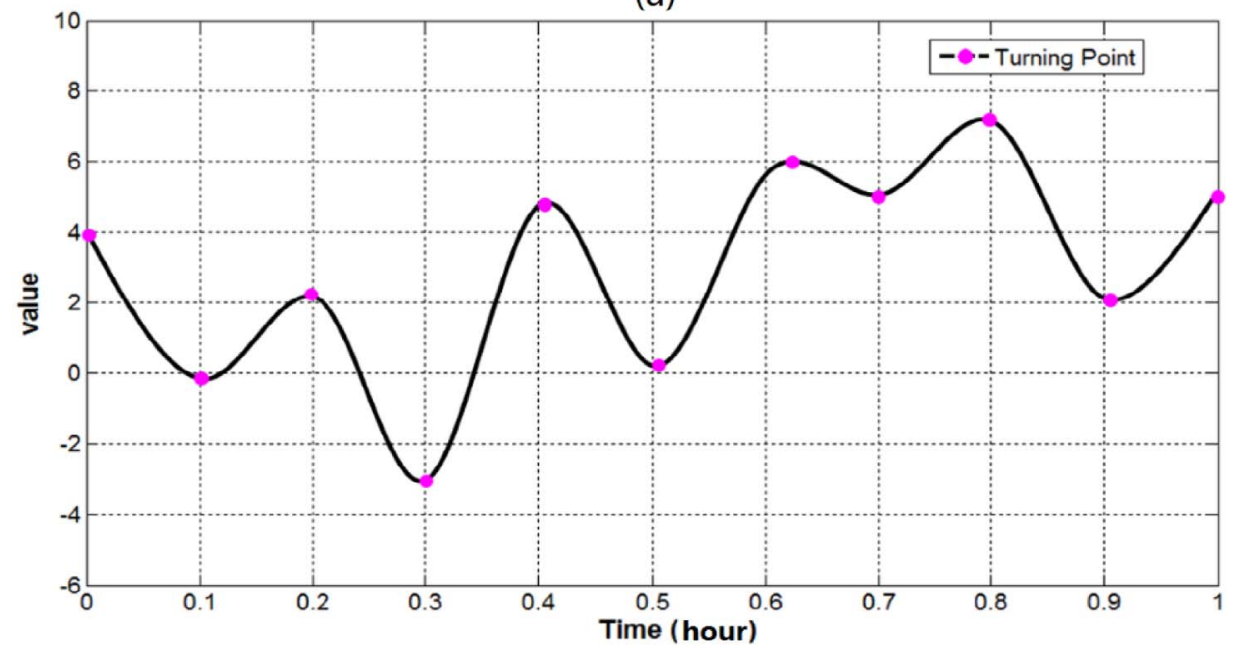

(b)

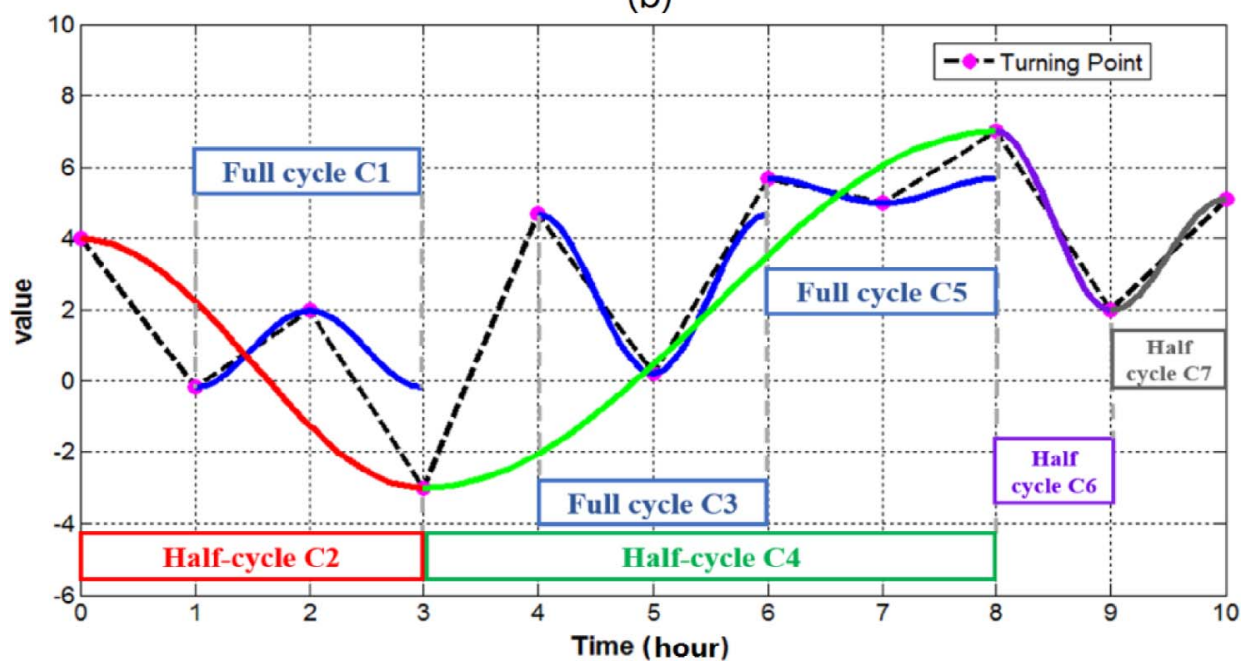

Fig. 8. (a) Example waveform. (b) Implementation the counting method on the example waveform.

Table 1

Summary of the cycle -counting result in the case study.

\begin{tabular}{llllllll}
\hline Labels (Cx) & C1 & C2 & C3 & C4 & C5 & C6 & C7 \\
\hline Amplitude & 2.2 & 7.0 & 4.5 & 10 & 0.7 & 5.0 & 3.1 \\
Number of cycles or half-cycles & 1 & 0.5 & 1 & 0.5 & 1 & 0.5 & 0.5 \\
Begin time of extracted sub-cycle & 1 & 0 & 4 & 3 & 6 & 8 & 9 \\
Cycle period (hour) $_{\text {C-rate }^{\mathrm{a}}}$ & 2 & 6 & 2 & 10 & 2 & 2 & 2 \\
& 2.2 & 2.3 & 4.5 & 2 & 0.7 & 5 & 3.1
\end{tabular}

a The discharge rate is in accordance with the discharge current and is often expressed as a C-rate by normalised means C-rate $=$ cycle amplitude/half-cycle period.

\subsection{Battery lifetime prediction method}

By integrating the battery with the SMES, the battery lifespan can be greatly improved, which is one of the key merits of the HESS. Qualitative analysis is not difficult as it can be observed from either experiment or simulation that the battery is protected by the SMES from the high-rate of discharge. In fact, many works $[26,39,40]$ have already studied battery life extension in the HESS scheme. However, in these works, it cannot be found as to what degree the battery lifetime is extended. In other words, the battery lifetime improvements in these studies are not quantified. However, in aiming to evaluate the HESS performance, quantitative analysis of the battery lifetime extension is very necessary. Therefore, to fill this gap, a battery lifetime prediction method is introduced in this paper.

\subsubsection{The rain-flow cycle-counting algorithm}

The rain-flow cycle-counting algorithm is usually used for analysing the fatigue data and was firstly used in metal fatigue estimation [2]. In this research, this method is used to extract the irregular charging and discharging cycles that the battery experienced in the MG. The cycle counting can be achieved by the following three steps, as shown in Fig. 7.

- Firstly, the data (battery depth of discharge that presents the battery charge/discharge cycles) are pre-processed by searching for adjacent data points with reverse polarity so that the local maxima and minima can be found and stored in a matrix.

- Secondly, compose full cycles by analysing the turning points and combine these sub-cycles to obtain full-cycles together with the summing up of the amplitudes and time durations.

- Thirdly, the number of cycles in varying amplitude and time duration are extracted and stored for later use.

A case study based on a simple waveform as shown in Fig. 8(a) is presented to illustrate the data processing of the rain-flow cyclecounting method.

As can be seen from Fig. 8(a) and (b), the exemplary signal is resolved and recombined by several cycles and half-cycles. Together with 


\section{EXPERIMENTS SETUP}

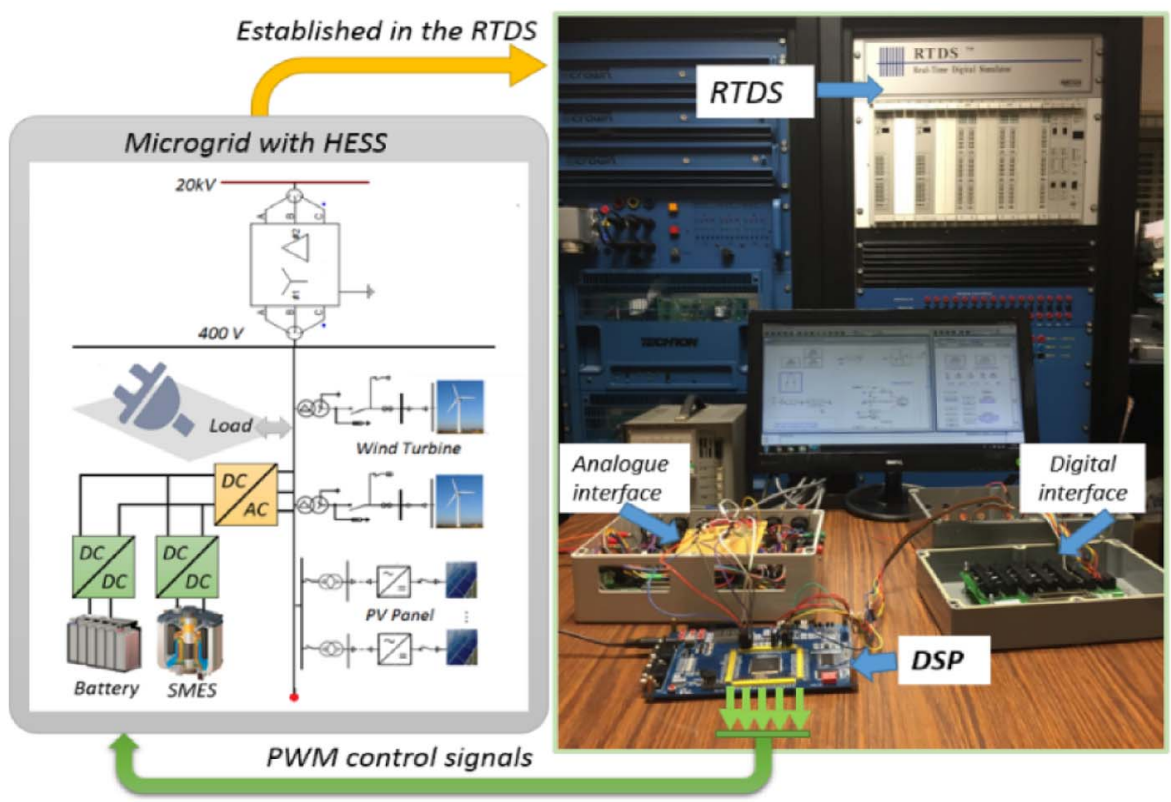

Fig. 9. The system configuration in the HIL test system.

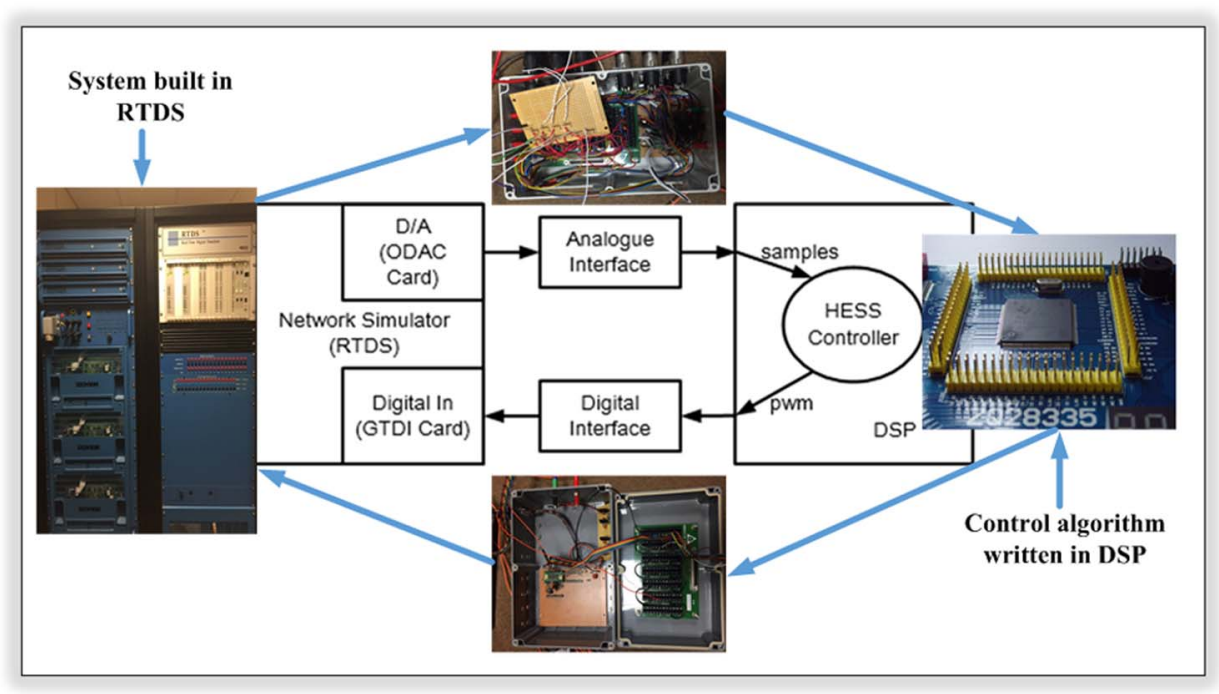

Fig. 10. Hardware-in-the-loop (HIL) testing system.

the cycle numbers, the amplitudes and time duration of these cycles are also stored in the algorithm. Table 1 summarizes the cycle-counting result.

\subsubsection{Battery lifetime prediction model}

In the hybrid energy storage scheme, the battery service time can be improved in two ways. Firstly, the small-scale charge and discharge cycles of the battery are reduced. Secondly, the battery is protected from the stinging currents. The battery lifetime model introduced in $[31,41]$ is able to calculate the effect of both the charge/discharge cycles and the discharging rate on battery degradation. Therefore, based on the cycle-counting results, this battery lifetime model is effectively applied to this study.

\section{Experiments setup}

As shown in Fig. 9, a HIL test system including the RTDS the analogue interface, the DSP and the digital interface, is developed to verify the optimal design of the SMES/battery HESS in this paper. The RTDS which has the real-time simulation capability and system flexibility is regarded as a more convincing and more accurate simulator than the other traditional simulation method [42-44]. Generally, it is very difficult to build a real MG in the laboratory environment. The RTDS uses parallel processing techniques on rack-mounted processors to maintain continuous real-time digital simulation of a power system [42]. The benefit of real-time operation means that the power system operates in its own closed loop and users are able to interact with the simulation in real time observing the effect of control actions. As a result, because of the real-time computing capability, the RTDS is regarded as a very effective tool for the experimental verification of power systems. The other significant advantage of the RTDS over the software simulation is that in the RTDS environment, all the signals and measurements are the real-time data, which make it possible to interface the external hardware/devices to the RTDS. In this study, a very 


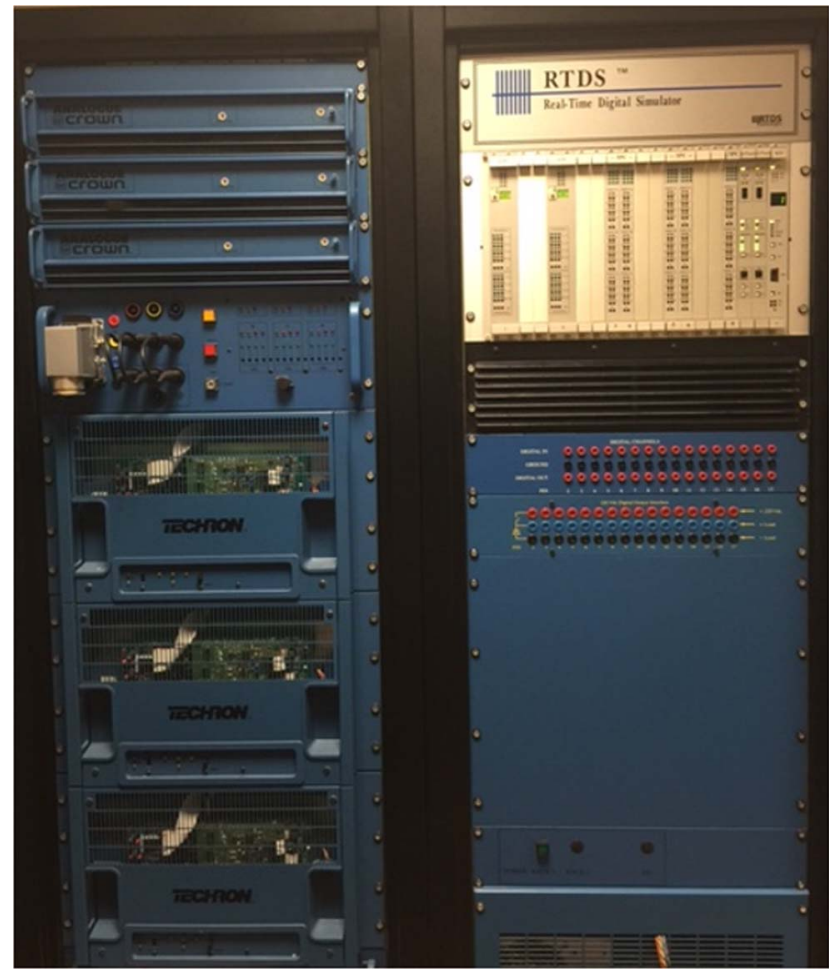

Fig. 11. Real-time digital simulator.

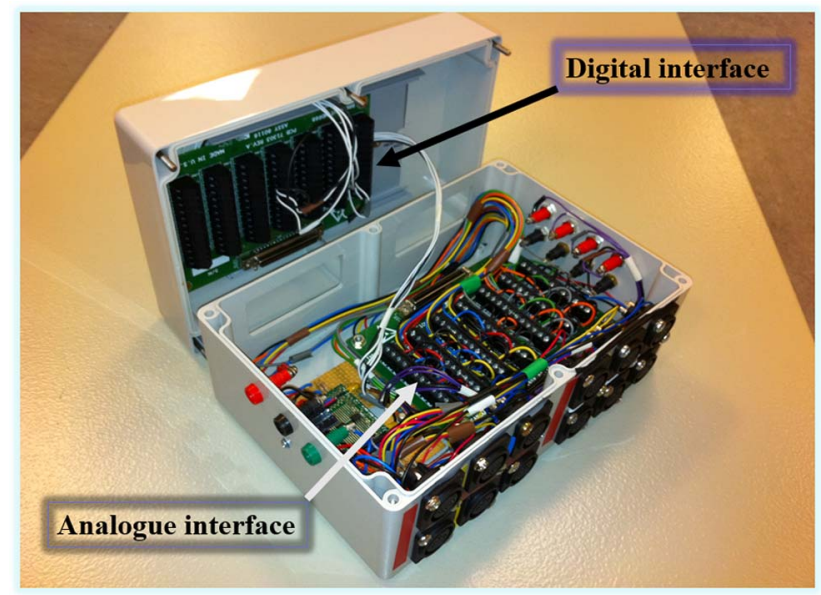

Fig. 12. The analogue and the digital interfaces used in the HIL circuit.

effective test platform is developed based on the RTDS and the hardware-in-loop circuit. In this topology, the RTDS will generate real-time measurements which will be sent to the DSP in the external circuit. The DSP will generate the physical control signals (PWM signals) which used to control the devices in the RTDS. As it can be seen from this process, the system is tested in the real-time, and the control algorithm is verified at an experimental level.

\subsection{Hardware in loop circuit}

Fig. 10 shows the HIL configuration which consists of the RTDS embed with the ODAC and GTDI cards, the analogue and the digital interfacing module and the DSP (TMS320F28335). The primary system is established and simulated in the RTDS. Analogue output is achieved through the onboard RTDS ODAC cards, which is responsible for converting the digital values from the RTDS to the analogue outputs. The analogue and the digital interfaces are used to connect the two hardware systems (the RTDS and the DSP). The function of the interfacing module is to process the output signals of the RTDS and the DSP and to make sure the input signals to each system are at the desired levels. The power management strategy and the HESS controller are implemented in the DSP. The DSP captures the analogue output signals from the ODAC card in RTDS and converts them to the digital signal by using the embedded ADC module. Then, based on the measurement data from the RTDS, the DSP generates the control signals. The control algorithms are also debugged in the DSP board. The PWM pulses generated by the DSP are read by the RTDS through its GTDI card.

\subsection{RTDS hardware}

The RTDS consists of two types of processing units, and they are named by the RTDS Technologies Inc. as 3PC and GPC cards. The "3" in the 3PC card means three digital signal processors (ADSP-21,062 SHARC Processors) and the clock frequency for each processor is about $40 \mathrm{MHz}$ The 3PC cards are usually arranged in parallel to increase the computing speed. The RTDS hardware as shown in Fig. 11 has ten 3PC cards, five in each rack. Five 3PC cards are used in this study. There are also two Giga processor cards (GPC) in the RTDS. The ODAC card and the GTDI card are embedded in the RTDS. The function of the ODAC card is to converter the digital values from the RTDS to the analogue outputs to the DSP. The GTDI card is responsible for reading the PWM signals from the DSP.

\subsection{Interface modules}

The interface modules are necessary to convert output signals between the RTDS and the DSP to acceptable input levels. As it can be seen from Fig. 12, two interfacing modules are needed in the HIL circuit, the analogue and the digital interfaces. The analogue interface module physically connected the analogue signal from the ODAC cards to the DSP ADC module. The ODAC card generates the analogue signal at $\pm 10 \mathrm{~V}$, whereas the input signal for the DSP 28335 peaks at $3.3 \mathrm{~V}$. Hence the interface circuit is needed to level the signals. The situation for the digital signal back circuit is similar to the analogue one that out signal from the DSP 28335 is driven by $3.3 \mathrm{~V}$ logic, whereas digital input port of the GTDI card takes $5 \mathrm{~V}$ logic. Therefore, in the signal back circuit, a digital interface is also needed.

\section{Results and discussions}

\subsection{Test of the new power-sharing control}

The net power disturbance which consists of the high-frequency power fluctuations and large power changes are shown in Fig. 13(a), and is used to test the performances of the previous and new controls. As shown in Fig. 13(b) and (c), both the previous filtration control and the new priority-based power sharing control are able to protect the battery from the short-term high power fluctuations and the SMESs are controlled to absorb most of the high-frequent components. However, at the large power changing point ( $4500 \mathrm{~s})$, the battery power changes much quicker from the positive to negative in the Fig. 13(b) than that in the Fig. 13(c). The abrupt power battery change in the previous control is very harmful to the battery lifetime. The new control method is able to avoid this situation since the battery charges and discharges according to the SMES current rather than the instantaneous power.

\subsection{Battery and SMES performance in the $M G$}

Fig. 14 gives the energy storage charge/discharge currents in battery only system (BOS) and the HESS with $6 \mathrm{~h}$ of simulation. It is obvious that the battery currents in the battery only system undergo extremely more polarity reversals and short-term charge/discharge processes than that in the HESS. The local peak battery currents in the 
(a)

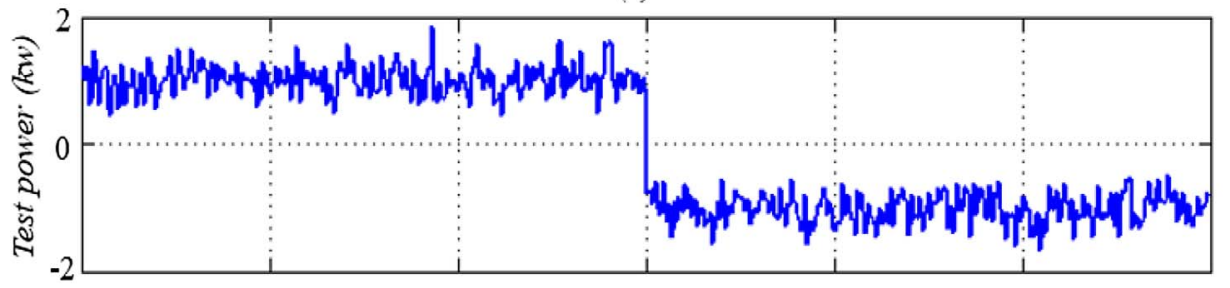

(b)



(c)

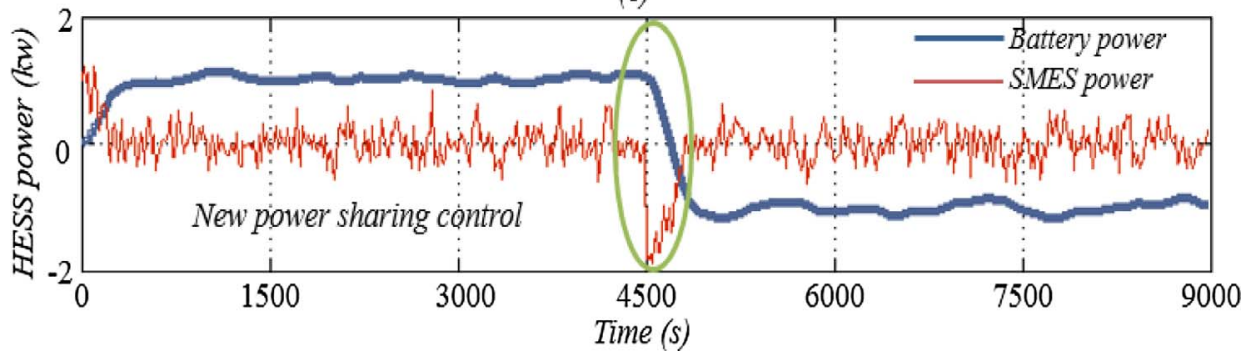

Fig. 13. (a) The test power, (b) HESS power using the previous filtration control and (c) HESS power using the new power sharing control.
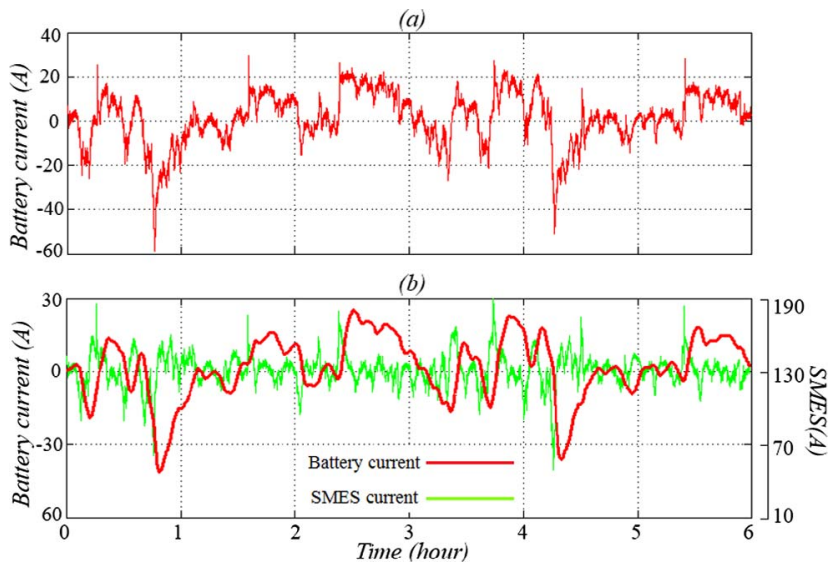

Fig. 14. (a) The battery current in the BOS system. (b) The battery current and the SMES current in the HESS.

Table 2

Battery lifetime qualification.

\begin{tabular}{llll}
\hline Scenarios & $\begin{array}{l}\text { Small-scale } \text { cycling } \\
\text { numbers in the } 6 \mathrm{~h}\end{array}$ & $\begin{array}{l}\text { Peak to peak } \\
\text { current }\end{array}$ & $\begin{array}{l}\text { Prediction of battery } \\
\text { lifetime }\end{array}$ \\
\hline BOS & 2364 & $83 \mathrm{~A}$ & 6.38 years \\
HESS & 27 & $62 \mathrm{~A}$ & 9.21 years \\
\hline
\end{tabular}

${ }^{\text {a }}$ Small-scale cycle means the cycle has the depth of discharge less that $10 \%$.

hybrid system are relatively smaller than that in the battery only system. Also, as it can be seen from Fig. 14(b), the SMES in the hybrid scheme has been successfully controlled to charge and discharge prior to the battery to the short-term power fluctuations.

\subsection{The battery lifetime extension qualification}

Based on the battery lifetime prediction method and the $6 \mathrm{~h}$ battery performance, Table 2 can be made to describe the lifetime improvement of the battery in the HESS.

In the hybrid energy storage design, the short-term high-frequency power fluctuation is absorbed by the SMES. Therefore, as shown in Table 2, the batteries undergo extensively fewer small-scale cycles that decrease from 2364 in the battery only system to 27 in the HESS. In addition, the larger peak to peak current in the battery only system means that the battery undergoes more stinging current without the cooperation of the SMES. Based on the battery lifetime model, battery lifespan in the BOS is predicted as 6.3 years whereas in the HESS it is 9.2 years resulting in $44.36 \%$ battery extension.

\section{Conclusion}

A SMES/battery hybrid energy storage system is proposed to be used in the microgrid to compensate the power fluctuations. A novel control method is developed and capable of sharing the instantaneous power between the SMES and battery based on the diffident characteristics of different energy storage units. In the new method, the SMES and the battery are operated in series position, and the power disturbances are firstly dealt by the SMES. The battery works as an energy buffer to the SMES and charges and discharges to maintain the SMES current in desired range. Due to the inductive characteristic of the SMES coil, the current flowing the SMES cannot change abruptly, which protects the battery from stinging currents. A HIL test platform consisting of the RTDS and the external control circuit is established to verify the hybrid design and the new control algorithm in experimental level. The results show that by using the new control, the battery in the HESS is protect from high power demand and rapid transient cycling. As a result, the battery lifetime can be extended by reducing cycling frequency and smoothing the sharp charge/discharge transient content. A quantitative evaluation of the battery lifetime extension in the HESS 
is also presented. In this study, the battery service time is improved by $44.36 \%$.

\section{References}

[1] Chaouachi A, Kamel RM, Andoulsi R, Nagasaka K. Multiobjective intelligent energy management for a microgrid. IEEE Trans Ind Electron 2013;60:1688-99.

[2] Li J, Xiong R, Yang Q, Liang F, Zhang M, Yuan W. Design/test of a hybrid energy storage system for primary frequency control using a dynamic droop method in an isolated microgrid power system. Appl Energy 2017;201:257-69.

[3] Yan J, Zhai Y, Wijayatunga P, Mohamed AM, Campana PE. Renewable energy integration with mini/micro-grids. Appl Energy 2017;201:241-4.

[4] Adhikari S, Li F. Coordinated Vf and PQ control of solar photovoltaic generators with MPPT and battery storage in microgrids. IEEE Trans Smart Grid 2014;5:1270-81.

[5] Zhang G, Cao Y, Cao Y, Li D, Wang L. Optimal energy management for microgrids with combined heat and power (CHP) generation, energy storages, and renewable energy sources. Energies 2017;10:1288.

[6] Jin X, Mu Y, Jia H, Wu J, Jiang T, Yu X. Dynamic economic dispatch of a hybrid energy microgrid considering building based virtual energy storage system. Appl Energy 2017;194:386-98.

[7] Hill CA, Such MC, Chen D, Gonzalez J, Grady WM. Battery energy storage for enabling integration of distributed solar power generation. IEEE Trans Smart Grid 2012;3:850-7.

[8] Li Z, Xu Y. Optimal coordinated energy dispatch of a multi-energy microgrid in gridconnected and islanded modes. Appl Energy 2017.

[9] Xiong R, Yu Q, Wang LY, Lin C. A novel method to obtain the open circuit voltage for the state of charge of lithium ion batteries in electric vehicles by using $\mathrm{H}$ infinity filter. Appl Energy 2017;207:346-53.

[10] Xiong $\mathrm{R}$, Zhang Y, He H, Zhou X, Pecht MG. A double-scale, particle-filtering, energy state prediction algorithm for lithium-ion batteries. IEEE Trans Ind Electron 2018;65(2):1526-38.

[11] Xiong R, Tian J, Mu H, Wang C. A systematic model-based degradation behavior recognition and health monitoring method for lithium-ion batteries. Appl Energy 2017;207:372-82

[12] Wei Z, Zhao J, Ji D, Tseng KJ. A multi-timescale estimator for battery state of charge and capacity dual estimation based on an online identified model. Appl Energy 2017.

[13] Wei Z, Meng S, Tseng KJ, Lim TM, Soong BH, Skyllas-Kazacos M. An adaptive model for vanadium redox flow battery and its application for online peak power estimation. J Power Sources 2017;344:195-207.

[14] Lujano-Rojas JM, Dufo-López R, Atencio-Guerra JL, Rodrigues EMG, Bernal-Agustín JL, Catalão JPS. Operating conditions of lead-acid batteries in the optimization of hybrid energy systems and microgrids. Appl Energy 2016;179:590-600.

[15] Nakayama T, Yagai T, Tsuda M, Hamajima T. Micro power grid system with SMES and superconducting cable modules cooled by liquid hydrogen. IEEE Trans Appl Supercond 2009;19:2062-5.

[16] Fagnard J-F, Crate D, Jamoye J-F, Laurent P, Mattivi B, Cloots R, et al. Use of a hightemperature superconducting coil for magnetic energy storage. J Phys Conf Ser 2006;43:829-32.

[17] Morandi A, Fiorillo A, Pullano S, Ribani PL. Development of a small cryogen-free MgB 2 test coil for SMES application. IEEE Trans Appl Supercond 2017;27:1-4.

[18] Wang Y, Song H, Yuan W, Jin Z, Hong Z. Ramping turn-to-turn loss and magnetization loss of a No-Insulation (RE) Ba2Cu3Ox high temperature superconductor pancake coil. J Appl Phys 2017;121:113903.

[19] Wang Y, Chan WK, Schwartz J. Self-protection mechanisms in no-insulation (RE) Ba2Cu3O x high temperature superconductor pancake coils. Supercond Sci Technol 2016;29:045007.

[20] Li J, Yang Q, Robinson F, Liang F, Zhang M, Yuan W. Design and test of a new droop control algorithm for a SMES/battery hybrid energy storage system. Energy 2017;118:1110-22.

[21] Ghaib K, Ben-Fares F-Z. A design methodology of stand-alone photovoltaic power systems for rural electrification. Energy Convers Manage 2017;148:1127-41.

[22] Chong LW, Wong YW, Rajkumar RK, Rajkumar RK, Isa D. Hybrid energy storage systems and control strategies for stand-alone renewable energy power systems. Renew Sustain Energy Rev 2016;66:174-89.

[23] Theo WL, Lim JS, Wan Alwi SR, Mohammad Rozali NE, Ho WS, Abdul-Manan Z. An MILP model for cost-optimal planning of an on-grid hybrid power system for an ecoindustrial park. Energy 2016;116:1423-41.

[24] Wieczorek M, Lewandowski M. A mathematical representation of an energy management strategy for hybrid energy storage system in electric vehicle and real time optimization using a genetic algorithm. Appl Energy 2017;192:222-33.

[25] Erdinc O, Vural B, Uzunoglu M. A wavelet-fuzzy logic based energy management strategy for a fuel cell/battery/ultra-capacitor hybrid vehicular power system. J Power Sources 2009;194:369-80.

[26] Ise T, Kita M, Taguchi A. A hybrid energy storage with a SMES and secondary battery. IEEE Trans Appl Supercond 2005;15:1915-8.

[27] Wang C, Xiong R, He H, Ding X, Shen W. Efficiency analysis of a bidirectional DC/ DC converter in a hybrid energy storage system for plug-in hybrid electric vehicles. Appl Energy 2016;183:612-22.

[28] Li J, Zhang M, Yang Q, Zhang Z, Yuan W. SMES/battery hybrid energy storage system for electric buses. IEEE Trans Appl Supercond 2016;26:1-5.

[29] Song Z, Hou J, Xu S, Ouyang M, Li J. The influence of driving cycle characteristics on the integrated optimization of hybrid energy storage system for electric city buses. Energy 2017;135:91-100.

[30] Zhou H, Bhattacharya T, Tran D, Siew TST, Khambadkone AM. Composite energy storage system involving battery and ultracapacitor with dynamic energy management in microgrid applications. IEEE Trans Power Electron 2011;26:923-30.

[31] Li J, Gee AM, Zhang M, Yuan W. Analysis of battery lifetime extension in a SMESbattery hybrid energy storage system using a novel battery lifetime model. Energy 2015;86:175-85.

[32] Yang Q, Le Blond S, Liang F, Yuan W, Zhang M, Li J. Design and application of superconducting fault current limiter in a multiterminal HVDC system. IEEE Trans Appl Supercond 2017;27:1-5.

[33] Yang Q, Le Blond S, Aggarwal R, Wang Y, Li J. New ANN method for multi-terminal HVDC protection relaying. Electr Power Syst Res 2017;148:192-201.

[34] Mohan V, Singh JG, Ongsakul W. An efficient two stage stochastic optimal energy and reserve management in a microgrid. Appl Energy 2015;160:28-38.

[35] Liu X, Wang P, Loh PC. A hybrid AC/DC microgrid and its coordination control. IEEE Trans Smart Grid 2011;2:278-86.

[36] Eghtedarpour N, Farjah E. Power control and management in a hybrid AC/DC microgrid. IEEE Trans Smart grid 2014;5:1494-505.

[37] Molina MG, Mercado PE. Power flow stabilization and control of microgrid with wind generation by superconducting magnetic energy storage. IEEE Trans Power Electron 2011;26:910-22.

[38] Han Y, Shen P, Zhao X, Guerrero JM. Control strategies for islanded microgrid using enhanced hierarchical control structure with multiple current-loop damping schemes. IEEE Trans Smart Grid 2015.

[39] Castaings A, Lhomme W, Trigui R, Bouscayrol A. Comparison of energy management strategies of a battery/supercapacitors system for electric vehicle under realtime constraints. Appl Energy 2016;163:190-200.

[40] Jia H, Mu Y, Qi Y. A statistical model to determine the capacity of battery-supercapacitor hybrid energy storage system in autonomous microgrid. Int $\mathrm{J}$ Electr Power Energy Syst 2014;54:516-24.

[41] Li J, Wang X, Zhang Z, Le Blond S, Yang Q, Zhang M, et al. Analysis of a new design of the hybrid energy storage system used in the residential m-CHP systems. Appl Energy 2017;187:169-79.

[42] Le Blond SP, Aggarwal RK. Design of adaptive autoreclosure schemes for $132 \mathrm{kV}$ network with high penetration of wind-Part I: real-time modeling. IEEE Trans Power Delivery 2012;27:1055-62.

[43] Zhao B, Xue M, Zhang X, Wang C, Zhao J. An MAS based energy management system for a stand-alone microgrid at high altitude. Appl Energy 2015;143:251-61.

[44] Avvari GV, Pattipati B, Balasingam B, Pattipati KR, Bar-Shalom Y. Experimental setup and procedures to test and validate battery fuel gauge algorithms. Appl Energy 2015;160:404-18. 